\title{
Evaluation of Miniature Implantable Antenna and Telemetry Link for Intracranial Pressure Monitoring
}

\author{
Konstantinos A. Psathas \\ Biomedical Simulations and Imaging Laboratory \\ National Technical University of Athens (NTUA) \\ Athens, Greece \\ kpsathas@biosim.ntua.gr
}

\author{
Konstantina S. Nikita \\ Biomedical Simulations and Imaging Laboratory \\ National Technical University of Athens (NTUA) \\ Athens, Greece \\ knikita@ece.ntua.gr
}

\begin{abstract}
Next generation (next-gen) Implantable Medical Devices (IMDs) attract significant scientific interest for early diagnosis, continuous monitoring and treatment. Wireless Intracranial Pressure (ICP) monitoring devices have been proposed for sub-dural and epidural implantation. The proposed devices overcome several limitations of existing wired techniques, providing great benefits for next-gen wireless ICP devices. In this work, we evaluate the telemetry link performance between a miniature biocompatible implantable antenna and an exterior dipole in MedRadio band. Moreover, we numerically assess the safety performance of the proposed antenna in terms of Specific Absorption Rate (SAR) and temperature increase in the surrounding head tissues.
\end{abstract}

Keywords-bioheat equation, implantable antenna, intracranial pressure monitoring, medical device radiocommunications (MedRadio) services, telemetry, temperature elevation, transmission coefficient.

\section{INTRODUCTION}

Recently research advances have enabled the use of millimeter and sub-millimeter scale Implantable Medical Devices (IMDs) that can be used as measurement/control devices (e.g. implanted sensors, drug infusion devices, artificial vision and organ control) or stimulators (cardioverters/defibrillators, cerebellar stimulators, other nerve stimulators, cochlear implants) [1-3]. Continuous or intermittent Intra-cranial Pressure (ICP) Monitoring consists the most feasible option for the management of patients with brain disorders or head injuries. Patients' ICP monitoring following a neurological surgery is required for proper treatment and adequate medication [4]. Limitations of the current ICP monitoring catheter-based devices have led to the introduction of wireless ICP monitoring prototypes with sufficient accuracy [5]. The use of wireless ICP monitoring devices can provide a feasible solution to the limitations of the current systems (e.g. patient discomfort, expensive equipment, wires and catheters originated from patient's head).

One of the most important aspects of an IMD is its antenna that enables the wireless communication of the implant with the exterior monitoring/control equipment. The globally available MedRadio band (formerly known as MICS band) [6], [7] has

This work has been co-financed by the European Union (European Social Fund, ESF) and national funds, under the project ARISTEIA DEM-II-MED ("Imnlantahle and Inoestihle Medical Devices (IIMDs)' Optimal-Performance- been proved to be the most favorable band for short-range inbody to out-of-body telemetry links due to the acceptable propagation through the human tissues and the relatively low noise portion of the spectrum. One of the major challenges of implantable antenna design is to achieve a wide- $10 \mathrm{~dB}$ bandwidth and provide a high performance telemetry link, while preserving patient safety and still maintaining miniature size for the antenna and consequently the IMD [8].

This work aims to evaluate the telemetry link performance between a miniature implantable antenna and an exterior dipole and assess the Specific Absorption Rate (SAR) and the temperature elevation and heat dissipation in head tissues caused by the imposed electromagnetic (EM) fields. In order to evaluate the telemetry link performance two scenarios are considered, with or without the presence of a wearable antenna relay, placed between the implantable antenna and the exterior dipole. Similar configurations have been used by the authors in [9] to evaluate a dual-band implantable antenna. In both scenarios, subdural implantation of the antenna in an anatomical human head model has been considered.

\section{MODELS AND METHODS}

\section{A. Antenna Model and Anatomical Head Phantom}

In this study, a slightly modified model of the implantable antenna which has been proposed and tested in-vitro and invivo by the authors in [10], is evaluated and shown in Fig. 1(a). The antenna features a planar inverted-F design philosophy with a radius of $7 \mathrm{~mm}$ (total volume of $293 \mathrm{~mm}^{3}$ ) and two meandered patch layers combined with a shorting pin for miniaturization purposes. The antenna uses a biocompatible ceramic alumina $96 \%$ material for substrate and superstrate layers and operates in the MedRadio band. Based on the findings of the authors in [11] alumina has been chosen as the most feasible way to maintain the antenna performance while making the original antenna biocompatible.

The relay antenna has been proposed in [8] (Fig. 1(b)) and designed to operate in the MedRadio and $2.4 \mathrm{GHz}$ Industrial Scientific and Medical (ISM) bands [6]. In this study, the performance of the antenna in the MedRadio band is examined. 
The antenna features a triangular patch plane and a shorting pin $(\mathrm{S})$, and exhibits a rectangular shape $(60 \mathrm{~mm} \times 70 \mathrm{~mm})$ with a total occupied volume of $6.72 \mathrm{~cm}^{3}$. Finally, a half-wavelength dipole antenna which operates in MedRadio is selected to serve as the exterior receiving antenna. All antennas are fed through 50-Ohm coaxial cables $(\mathrm{F})$.

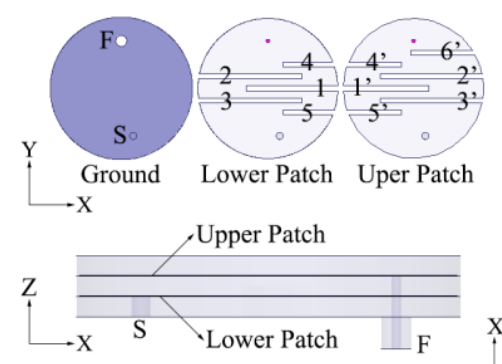

(a)
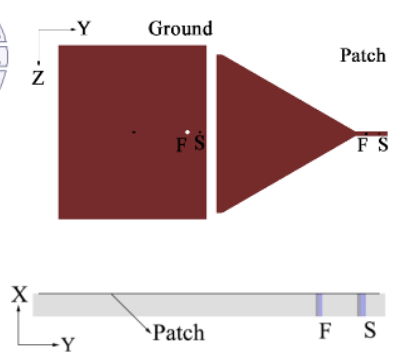

(b)

Fig. 1. (a) Miniature antenna model [10], (b) relay antenna used between the implantable and exterior antennas [8].

Duke anatomical head model with compressed ears (part of the Virtual Population by IT'IS) (Fig. 2) [12], which includes 45 types of biological tissues is used in the simulations. The anatomical head model is centered in the XY plane and the antenna is placed at a depth of $3 \mathrm{~mm}$ from the head surface.

\section{B. Numerical Methods}

Finite Difference Time Domain (FDTD) simulations are carried out using the SEMCAD X software by SPEAG [13]. For electromagnetic simulations, a minimum spatial resolution of $72.5 \times 93.4 \times 105.8 \mu \mathrm{m}^{3}$ and maximum spatial resolution of 10.5 $\mathrm{x} 10.4 \mathrm{x} 9.6 \mathrm{~mm}^{3}$ in the $\mathrm{x}, \mathrm{y}$, and $\mathrm{z}$ directions is chosen for MedRadio band. The max step satisfies the FDTD spatial step constraint and is conservatively chosen in an attempt to reduce dispersion errors generated by the non-uniformity of the grid. Gaussian sources (pulse width of 40 periods is chosen to achieve a steady state) are used for broadband simulations.

Thermal simulations are carried out based on the Pennes' Bioheat Equation (PBE) [14]:

$$
\rho c \frac{\partial T}{\partial t}=\nabla \cdot(k \nabla T)+\rho S+\rho_{b} c_{b} \rho \omega\left(T-T_{b}\right)
$$

where, $k$ is the tissue thermal conductivity, $S$ is the SAR, $\omega$ is the perfusion rate, $\rho$ is the density of the medium, and $\rho_{b}, c_{b}$, and $T_{b}$ are the mass density, specific heat capacity and temperature of the blood. The grid size is set to $1 \mathrm{~mm}$ and $5 \mathrm{~mm}$ for minimum and maximum spatial resolution respectively on $\mathrm{x}, \mathrm{y}$, and $\mathrm{z}$ axis. All the required parameters for the head tissues are defined according to [15]. The boundary condition at the interface of skin tissue and the air, is defined by the equation:

$$
k \frac{d T}{d n}+h\left(T-T_{\text {outside }}\right)=F_{\text {boundary }}
$$

where, $\mathrm{T}_{\text {outside }}$ describes the room temperature and is set to $25^{\circ} \mathrm{C}$, $\mathrm{h}$ is the heat transfer coefficient between the head skin and the air and is defined as $5 \mathrm{~W} / \mathrm{m}^{2} / \mathrm{K}$, while heat flux is set to 0 $\left(F_{\text {boundary }}\right)$. The heat transfer coefficient is used to emulate the thermoregulation mechanism and the sweating effect.

\section{RESULTS AND DISCUSSION}

\section{A. Simulation Setup and Antennas Performance}

The simulation set-ups are shown in Fig. 2. The upper surface of the implantable antenna is placed $3 \mathrm{~mm}$ under the external surface of the head model while the center of the antenna ground plane coincides with the origin of the coordinates system, $\mathrm{O}(0,0,0))$. The exterior receiving dipole is placed along the z-axis, as shown in Fig. 2, and can move across the $\mathrm{Y}$-axis. The relay antenna is placed as shown in Fig. 2(b), at a distance $\mathrm{d}_{3}=80 \mathrm{~mm}$ from $\mathrm{O}(0,0,0)$ across $\mathrm{x}$-axis.

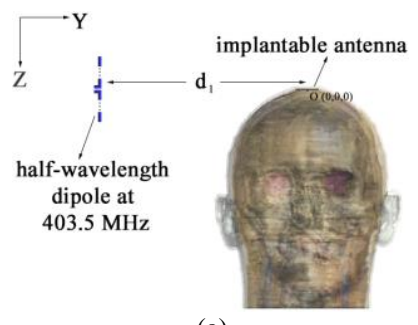

(a)

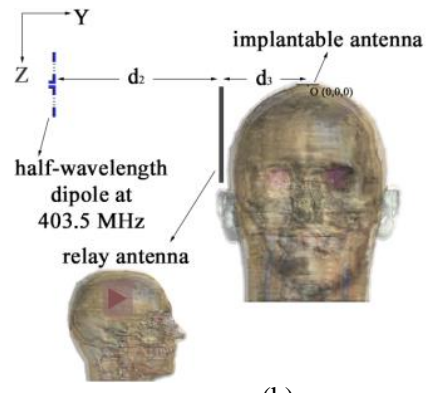

(b)
Fig. 2. (a) Simulation set-ups: (a) without the relay antenna, and (b) with the relay antenna.

The reflection coefficient $\left(\left|S_{11}\right|\right)$ frequency response of the implantable and relay antennas is illustrated in Fig. 3. The implantable antenna resonates at $403 \mathrm{MHz}$ with a bandwidth of $26.5 \mathrm{MHz}$, while the wearable antenna resonates at $406.16 \mathrm{MHz}$ and with a bandwidth of $6.4 \mathrm{MHz}$. The implantable antenna exhibits a directional and asymmetrical radiation pattern (Fig. 4(a)). Maximum far-field gain $\left(\mathrm{G}_{\max }\right)$ values at $403.5 \mathrm{MHz}$ are computed as $-41.32 \mathrm{dBi}$. Asymmetry of the radiation pattern is due to the inhomogeneous dielectric structure of the anatomical head. The relay antenna exhibits an almost omni-directional radiation pattern (Fig. 4(b)). Maximum far-field gain $\left(\mathrm{G}_{\max }\right)$ value at $403.5 \mathrm{MHz}$ is equal to $-20.04 \mathrm{dBi}$.

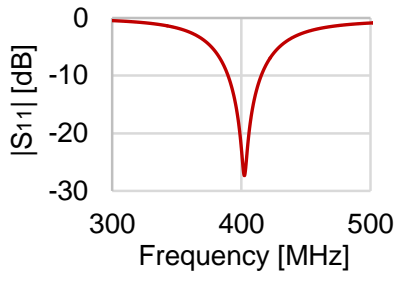

(a)

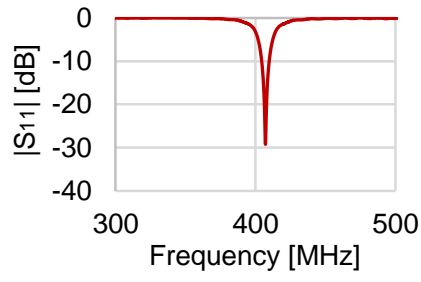

(b)
Fig. 3. Resonance (reflection coefficient frequency response) performance of the (a) implantable and (b) relay antenna in MedRadio band.

Next, safety performance in terms of SAR of the implantable and relay antennas is evaluated. The exhibited maximum SAR values averaged over $1 \mathrm{~g}\left(\mathrm{SAR}_{1 \mathrm{~g} \text {-max }}\right)$ and $10 \mathrm{~g}\left(\mathrm{SAR}_{10 \mathrm{~g} \text {-max }}\right)$ of tissue and the corresponding maximum input power to the implantable and relay antennas according to the latest IEEE standards are summarized in Table I.

Thermal simulations are carried out to assess the temperature elevation and spatial heat dissipation in human head tissues. Heat Generation Rate (HGR) of human tissues is not considered in this study, as we numerically assess the temperature elevation 

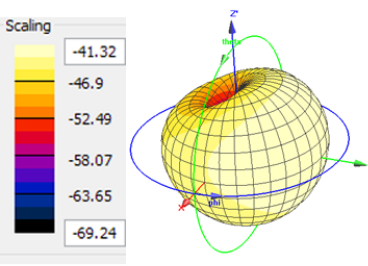

(a)
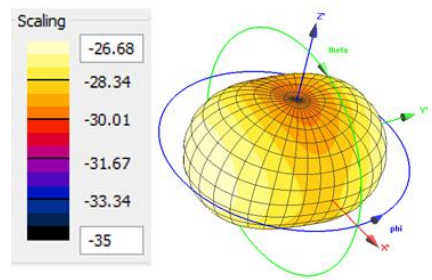

(b)
Fig. 4. Radiation (3D far-field radiation pattern) performance of the (a) implantable and (b) relay antenna in MedRadio band.

due to the operation of the implantable antenna. Input power of the implantable antenna is set to $20.9 \mathrm{~mW}$ according to the Table I in order to ensure the compliance with the latest IEEE C.95.1-2005 guidelines. The total thermal simulation time has been set to $2400 \mathrm{sec}$ (40 $\mathrm{min})$.

TABLE I. SAFETY PERFORMANCE OF THE IMPLANTABLE AND RELAY ANTENNA AT $403.5 \mathrm{MHz}$

\begin{tabular}{|l|c|c|c|c|}
\hline \multirow{2}{*}{ Antenna } & \multicolumn{4}{|c|}{ Safety Performance } \\
\cline { 2 - 5 } & $\begin{array}{c}\boldsymbol{S A R}_{\boldsymbol{1 g} \text {-max }} \\
{[\mathbf{m} \boldsymbol{W} / \boldsymbol{g}]}\end{array}$ & $\begin{array}{c}\boldsymbol{S A R}_{\boldsymbol{1 0 g} \text {-max }} \\
{[\mathbf{m} \boldsymbol{W} / \mathbf{g}]}\end{array}$ & $\begin{array}{c}\boldsymbol{P}_{\max -1999} \\
{[\mathbf{m} \boldsymbol{W}]}\end{array}$ & $\begin{array}{c}\boldsymbol{P}_{\max -2005} \\
{[\mathbf{m} \boldsymbol{W}]}\end{array}$ \\
\hline Miniature & 435 & 95.6 & 3.67 & 20.9 \\
\hline Wearable & 4.25 & 1.63 & 376.47 & 815 \\
\hline
\end{tabular}

The transient results are summarized in Fig. 4. Point A (-1.7 $\mathrm{mm},-5.8 \mathrm{~mm}, 0.7 \mathrm{~mm}$ ) has been chosen to monitor temperature elevation over time as it is the point that the maximum temperature $\left(0.887{ }^{\circ} \mathrm{C}\right)$ is observed during the simulation. Another spot, point B $(0 \mathrm{~mm}, 0 \mathrm{~mm}, 22 \mathrm{~mm})$, has been chosen to monitor temperature elevation over time in the area of the grey matter of the human brain as this area is critical for the physicians. A maximum temperature of $0.0135{ }^{\circ} \mathrm{C}$ has been observed in Point B, which is significantly lower than the one observed at Point A (skin tissue). Finally, spatial temperature distribution in axial, coronal and sagittal views is summarized in Fig. 5.

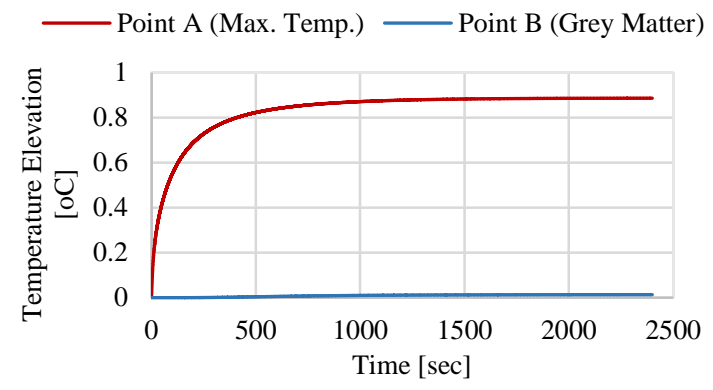

Fig. 5. Temperature elevation over time for MedRadio at the pre-defined point $\mathrm{A}(-1.7 \mathrm{~mm},-5.8 \mathrm{~mm}, 0.7 \mathrm{~mm})$ and $\mathrm{B}(0 \mathrm{~mm}, 0 \mathrm{~mm}, 22 \mathrm{~mm})$.

\section{B. Telemetry Link Evaluation}

In this study, half-duplex communication is studied between the implantable antenna and the exterior half-wavelength dipole, without (Fig. 2(a)) and with (Fig. 2(b)) the use of the intermediate relay antenna. As part of this study, the relay

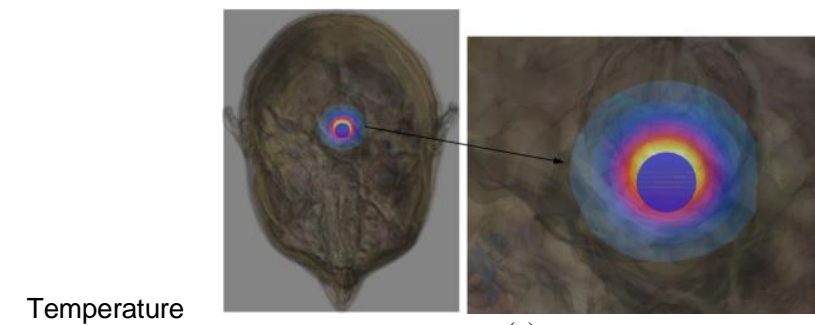

(a)
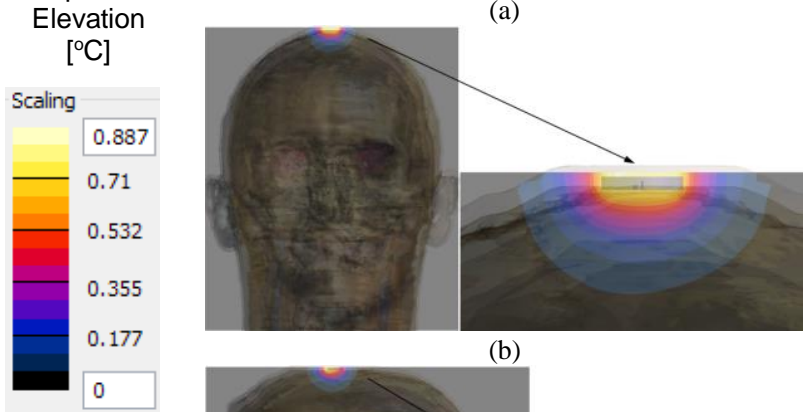

(b)

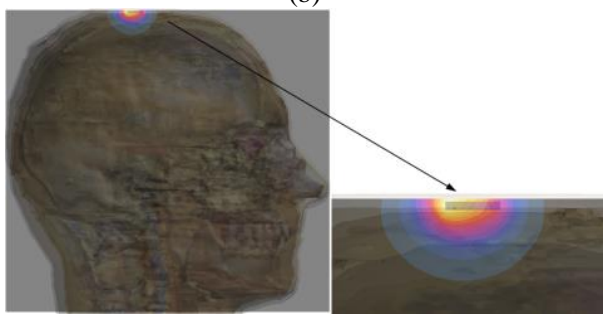

(c)

Fig. 6. Spatial temperature distribution in (a) axial (XY plane), (b) coronal (XZ plane), and (c) sagittal (YZ plane) view in MedRadio band.

antenna is placed in close proximity to the head model as shown in Fig. 2(b). The position and orientation of the relay antenna has been adopted here as a proof of concept for the assessment of the telemetry link performance. Since the relay antenna is printed on a flexible substrate material, the antenna can be conformed in the shape of a wearable band or a patch and placed on the human head. The conformance of the relay antenna, under the aforementioned realistic scenarios, doesn't imply a significant change in the antenna performance, but a slight retuning of the antenna design parameters may be required to maintain its performance.

In the first scenario (Fig. 2(a)), a telemetry link is established directly between the implantable antenna and the exterior dipole in the MedRadio band. The corresponding transmission coefficient frequency response for distances from 1 to $5 \mathrm{~m}$ is shown in Fig. 7. Considering that the typical receiver sensitivity of the Microsemi ZL70120 MICS-Band RF Base Station Module [16] is equal to $-91 \mathrm{dBm}$, the telemetry link is found to be reliable up to a distance of $5 \mathrm{~m}$ at $403.5 \mathrm{MHz}$ between the antennas for an input power of $0.0257 \mathrm{~mW}(-16 \mathrm{dBm})$.

In the second scenario (Fig. 2(b)), the wearable antenna is placed between the implantable and the exterior antenna and acts as a relay between them. In order to evaluate the telemetry link the transmission coefficient $\left(\left|\mathrm{S}_{21}\right|\right)$ between the implantable and wearable antenna is calculated at the fixed distance of Fig. 2(b) and is found to be equal to $-63.3 \mathrm{dBm}$ at $403.5 \mathrm{MHz}$. Assuming the same receiver sensitivity of $-91 \mathrm{dBm}$ in the relay, 


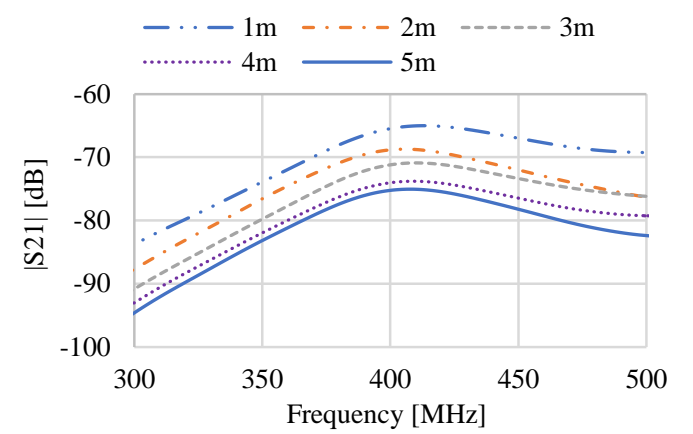

Fig. 7. Transmission coefficient $\left(\left|S_{21}\right|\right)$ frequency response for distances from 1 to $5 \mathrm{~m}$ between the implantable antenna and the exterior dipole without the presence of the relay antenna.

this value of $\left|\mathbf{S}_{21}\right|$ enables the reduction of the implantable antenna's input power to $0.0017 \mathrm{~mW}(-27.7 \mathrm{dBm})$ for reliable communication between the implant and the relay antenna. The $\left|S_{21}\right|$ between the relay antenna and the exterior dipole is then studied. In this case, and for a receiver sensitivity of $-91 \mathrm{dBm}$, the input power of the relay antenna can be limited to $0.43 \mathrm{~mW}$ (or $-3.7 \mathrm{dBm}$ ) for reliable communication at a distance of $5 \mathrm{~m}$. The value of the relay antenna's input power is significantly lower than the maximum input power $\mathrm{P}_{\max -2005}$ which was calculated in Table I, ensuring the extremely low exposure of the human head tissues to EM fields. Based on the above calculations, the use of the relay antenna allows for a $93 \%$ reduction in the input power of the implanted antenna.

\section{CONCLUSION}

In this work, we performed EM and thermal simulations to numerically assess the proposed implantable and wearable antenna performance and their co-existence in an integrated system for ICP monitoring evaluating the telemetry link performance. The proposed implantable antenna is a slightly modified biocompatible version (printed in the biocompatible alumina $96 \%$ material) of the original antenna which has been measured in-vitro and in-vivo in previous studies of the authors.

Resonance, radiation and safety performance in terms of SAR and temperature elevation of the miniature biocompatible implantable antenna was evaluated. Moreover, the telemetry link between the implantable antenna and an exterior dipole, with and without the presence of a wearable relay antenna was examined. It was demonstrated that the use of the relay as an integrated part of an integrated ICP monitoring system results in a reduction by $93 \%$ of the required power of the implanted antenna to establish a reliable telemetry link with the exterior monitoring/control device. As a result, the reduction of the implantable antenna's input power leads to significant benefits in terms of both energy efficiency of the implant and the EM exposure of the head tissues.

\section{ACKNOWLEDGMENT}

The authors would like to thank Schmid \& Partner Engineering AG (SPEAG) for providing the license for SEMCAD-X software through SEMCAD X for Science agreement.

\section{REFERENCES}

[1] A. Kiourti, K. A. Psathas, and K. S. Nikita, "Implantable and ingestible medical devices with wireless telemetry functionalities: A review of current status and challenges," Bioelectromagnetics, vol. 35, pp. 1-15, 2014.

[2] K. Jaehoon and Y. Rahmat-Samii, "Implanted antennas inside a human body: simulations, designs, and characterizations," Microwave Theory and Techniques, IEEE Transactions on, vol. 52, pp. 1934-1943, 2004.

[3] T. Karacolak, A. Z. Hood, and E. Topsakal, "Design of a Dual-Band Implantable Antenna and Development of Skin Mimicking Gels for Continuous Glucose Monitoring," Microwave Theory and Techniques, IEEE Transactions on, vol. 56, pp. 1001-1008, 2008.

[4] P. Marik, K. Chen, J. Varon, R. Fromm Jr, and G. L. Sternbach, "Management of increased intracranial pressure: A review for clinicians," The Journal of Emergency Medicine, vol. 17, pp. 711-719, 7// 1999.

[5] U. Kawoos, M. R. Tofighi, R. Warty, F. A. Kralick, and A. Rosen, "InVitro and In-Vivo Trans-Scalp Evaluation of an Intracranial Pressure Implant at $2.4 \mathrm{GHz}, "$ Microwave Theory and Techniques, IEEE Transactions on, vol. 56, pp. 2356-2365, 2008.

[6] FCC. Washington, D.C., USA, Federal Communications Commission 2012 [Online] Available: http://www.fcc.gov.

[7] A. Kiourti, M. Christopoulou, and K. S. Nikita, "Performance of a novel miniature antenna implanted in the human head for wireless biotelemetry," in Antennas and Propagation (APSURSI), 2011 IEEE International Symposium on, 2011, pp. 392-395.

[8] A. Kiourti, J. R. Costa, C. A. Fernandes, and K. S. Nikita, "Title," unpublished|.

[9] K. A. Psathas and K. S. Nikita, "Temperature Elevation Produced by Miniature Implantable Antennas for Intracranial Pressure Monitoring," presented at the URSIGASS 2014, Beijing, China, 2014.

[10] A. Kiourti, K. A. Psathas, P. Lelovas, N. Kostomitsopoulos, and K. S. Nikita, "In Vivo Tests of Implantable Antennas in Rats: Antenna Size and Intersubject Considerations," Antennas and Wireless Propagation Letters, IEEE, vol. 12, pp. 1396-1399, 2013.

[11] K. A. Psathas, A. Kiourti, and K. S. Nikita, "Biocompatibility of Implantable Antennas: Effects in Design and Performance," presented at the 8th European Conference on Antennas and Propagation (EuCAP 2014), The Hague, The Netherlands, 2014.

[12] A. Christ, W. Kainz, E. G. Hahn, K. Honegger, M. Zefferer, E. Neufeld, et al., "The Virtual Family--development of surface-based anatomical models of two adults and two children for dosimetric simulations," Phys Med Biol, vol. 55, pp. N23-38, Jan 212010.

[13] SEMCAD X, Schmid \& Partner Engineering AG (SPEAG), version 14.8 Aletsch. [Online]. Available: http://www.speag.com/products/semcad/overview/.

[14] S. Gabriel, R. W. Lau, and C. Gabriel, "The dielectric properties of biological tissues: III. Parametric models for the dielectric spectrum of tissues," Phys Med Biol, vol. 41, pp. 2271-93, Nov 1996.

[15] S. Gabriel, R. W. Lau, and C. Gabriel, "The dielectric properties of biological tissues: II. Measurements in the frequency range $10 \mathrm{~Hz}$ to 20 GHz," Phys Med Biol, vol. 41, pp. 2251-69, Nov 1996.

[16] "Microsemi, ZL70120 MICS-Band RF Base Station Module [Online] Available:

http://www.microsemi.com/documentportal/doc download/125431-zl70102-full-datasheet." 